\title{
Postocclusive Hyperemia Measured with Laser Doppler Flowmetry and Transcutaneous Oxygen Tension in the Diagnosis of Primary Raynaud's Phenomenon: A Prospective, Controlled Study
}

\author{
Paweł Maga, ${ }^{1}$ Brandon Michael Henry, ${ }^{2}$ Elizabeth K. Kmiotek, ${ }^{2}$ Iwona Gregorczyk-Maga, ${ }^{3}$ \\ Paweł Kaczmarczyk, ${ }^{1}$ Krzysztof A. Tomaszewski, ${ }^{2}$ and Rafał Niżankowski ${ }^{1}$ \\ ${ }^{1}$ Department of Angiology, Jagiellonian University Medical College, 8 Skawińska Street, 31-066 Krakow, Poland \\ ${ }^{2}$ Department of Anatomy, Jagiellonian University Medical College, 12 Kopernika Street, 31-034 Krakow, Poland \\ ${ }^{3}$ Department of Pediatric Dentistry, Institute of Dentistry, Jagiellonian University Medical College, 4 Montelupich Street, \\ 31-155 Krakow, Poland \\ Correspondence should be addressed to Krzysztof A. Tomaszewski; krtomaszewski@gmail.com
}

Received 19 July 2016; Accepted 21 November 2016

Academic Editor: Francesco Del Galdo

Copyright (C) 2016 Paweł Maga et al. This is an open access article distributed under the Creative Commons Attribution License, which permits unrestricted use, distribution, and reproduction in any medium, provided the original work is properly cited.

\begin{abstract}
The aim of this study was to measure the sensitivity and specificity of transcutaneous oxygen tension and postocclusive hyperemia testing using laser Doppler flowmetry in patients with primary Raynaud's phenomenon. One hundred patients and one hundred controls were included in the study. Baseline microvascular blood flow and then time to peak flow following occlusion were measured using laser Doppler flowmetry. Afterwards, the transcutaneous oxygen tension was recorded. The sensitivities of baseline microvascular blood flow, postocclusive time to peak flow, and transcutaneous oxygen tension were 79\%, 79\%, and 77\%, respectively. The postocclusive time peak flow had a superior specificity of $90 \%$ and area under the curve of 0.92 as compared to $66 \%$ and 0.80 for baseline microvascular flow and $64 \%$ and 0.76 for transcutaneous oxygen tension. Time to postocclusive peak blood flow measured by laser Doppler flowmetry is a highly accurate test for differentiating patients with primary Raynaud's phenomenon from healthy controls.
\end{abstract}

\section{Introduction}

Raynaud's phenomenon (RP) is a disorder characterized by episodic vasospastic attacks of the small blood vessels that supply the skin of the digits that is precipitated by exposure to cold or emotional stress. The syndrome takes its name from Raynaud, who first described the disorder in his 1862 doctoral thesis [1]. The course of a typical RP attack is an initial ischemic phase manifested by blanching or cyanosis, followed by a rapid reflow of blood marked by digital erythema (reactive hyperemia).

Vasoconstriction and cutaneous color changes following exposure to cold stimuli are a normal physiological response and the pathomechanism which distinguishes RP from this normal reaction is still unclear $[2,3]$. However, evidence suggests that the mechanism in primary RP may involve an overexpression or hyperactivity of postsynaptic alpha-2 adrenergic receptors in the smooth muscle of digital vessels $[4,5]$.

To date, diagnosis is based on the clinical picture of reversible changes in color of the digits and discomfort in response to cold or emotional stress [6,7]. However, diagnosis of RP based on observation or patient reports alone makes it difficult to distinguish patients with primary RP from those who either are simply sensitive to cold temperatures or developed secondary RP related to various conditions, particularly scleroderma, in which RP symptoms are often observed. Cold challenge tests are generally not used clinically, may cause patient injury, and have low sensitivity, specificity, and reproducibility due to individual variation 
in cutaneous blood flow patterns under normal physiologic conditions [6-8]. A clear systematic approach to objective diagnosis of RP has yet to be elucidated.

While primary RP is considered a benign condition, studies have indicated that patients suffering from primary RP experience a significant decrease in their quality of life $[9,10]$. Furthermore, it is well established that patients with RP are more likely to develop other rheumatic diseases in the future. For example, in Buerger's disease, RP is a common initial symptom and is present in up to $40 \%$ of patients with the condition $[11,12]$. As such, a more accurate and objective diagnosis of patients with primary RP would allow for more careful evaluation and management of the patient's condition by clinicians.

Much emphasis has been placed on the study of microcirculation in patients with RP in the aim of understanding the vascular underpinnings of the condition, as well as developing an efficient method for diagnosis. A number of different techniques have previously been employed to study skin microcirculation such as capillaroscopy $[13,14]$, cold challenge tests [15-17], laser Doppler flowmetry (LDF) [18-22], thermography [16, 23, 24], and measurement of transcutaneous oxygen tension [25-27]. However, to date, no diagnostic test has been universally implemented for aiding in the diagnosis of primary RP [6].

LDF is a valuable, noninvasive method of analyzing microcirculatory changes in RP $[18-22,28]$. It measures the flux of blood cells in a designated area, making it well suited to monitor transient alterations in skin circulation, as well as determine general perfusion [29-32]. LDF has good reproducibility, especially under standardized provocations $[33,34]$ and, therefore, has been recommended as a diagnostic tool in patients with suspected RP $[19,28]$. However, LDF has not been adapted for widespread clinical use and is viewed by some medical practitioners as inappropriate due to difficulties with implementation and to having a questionable utility [6].

Some of the difficulties with implementation of LDF as a diagnostic tool are related to its single point of measure [35]. These include the sensitivity of the probe to tissue heterogeneity and movement artefacts. Some of the issues with implementation can be overcome through the use of dynamic testing, such as postocclusive reactive hyperemia, with a probe that is, in a fixed position, firmly attached to skin [35]. Newer laser Doppler techniques, such as laser Doppler imaging (LDI), which measures flux over an area instead of a single point, are being explored for the use in patients with RP and have thus far reported interesting results [36-38]. However, the newer LDI equipment is more expensive and is not yet readily accessible for many clinicians [35].

Transcutaneous oxygen tension is another noninvasive method which has demonstrated efficacy in evaluating perfusion in patients with $\mathrm{RP}[27,39]$. Baseline $\mathrm{TcPO}_{2}$ has been found to be lower in patients with disturbances in nutritive skin perfusion, such as in RP [27].

Transcutaneous oxygen tension and LDF measures may provide more certainty in objectively assessing subjects with RP and can be an important addition to the currently available diagnostic paradigms and thus merits further investigation. The aim of the present study was to assess the sensitivity and specificity of $\mathrm{TcPO}_{2}$ and postocclusive hyperemia measured with LDF in patients with primary RP.

\section{Materials and Methods}

2.1. Patients. The study sample was composed of 100 patients (75 women and 25 men) aged 17 to 49 (mean \pm SD $24.97 \pm$ 6.37) with a diagnosis of primary RP, as defined by LeRoy and Medsger criteria [40] and 100 controls aged 19 to 27 (mean \pm SD $23.12 \pm 2.38$ ) with the same proportion of men and women as the study sample. All participants had their titers of antinuclear antibodies and rheumatoid factor tested and underwent an evaluation of their thyroid function. For inclusion in the study, all the tested parameters had to be within normal limits and not reveal any autoimmune or thyroid dysfunctions. The exclusion criteria for the study included a history of smoking (within the past six months), diabetes, hypertension, or any regular medication use, including oral anticontraceptives, hormone replacement therapy, and nicotine replacement therapy. No participant had any trophic changes to the fingers, and participants were asked not to remove any periungual tissues two weeks prior to the study. All participants were asked to refrain from consumption of any caffeinated or alcoholic beverages prior to the examination. No participant was wearing nail varnish during the testing. Furthermore, capillaroscopic tests were performed to ensure that no morphological abnormalities were present in the tested areas, as such findings would be suggestive of secondary RP [41]. Investigators conducting further tests ( $\mathrm{LDF}$ and $\mathrm{TcPO}_{2}$ measurements) were blind as to whether the subject belonged to the RP patient group or to the control group.

2.2. Tests. Each test was carried out in a room with an increased sound absorption at a temperature of 21-23 degrees Celsius. Testing was preceded by a 15 -minute resting period, during which the patient sat in a comfortable position. Finger skin temperature was measured using ELLAB ctd 85 system (Copenhagen, Denmark) with the ELLA PRC A probe, calibrated per the manufacturer's guidelines. The probe was positioned on lateral aspect of the distal phalanx and was stabilized using the pressure cuff of the occlusion test (Figure 1). Baseline microvascular blood flow was then measured using the LDF apparatus for a duration of 5 minutes. Following the baseline assessment, LDF postocclusion hyperemia time to peak blood flow and $\mathrm{TcPO}_{2}$ values were measured. For the purposes of this paper, baseline microvascular blood flow measured in arbitrary perfusion units (PU) will be referred to as bMBF, and the postocclusion time to peak blood flow measured in seconds will be referred to as tpMBF.

2.3. $L D F$. LDF measurements were made using the Periflux4001 Master apparatus (Perimed AB, Jarfalla, Sweden) calibrated per the manufacturer's guidelines with the Capy Flow software package to register and analyze the results. The apparatus was equipped with a probe of standard fiber separation $(0.25 \mathrm{~mm})$ and a $780 \mathrm{~nm}$ wavelength laser with a measuring depth of $0.5-1.0 \mathrm{~mm}$. The probe was stabilized 


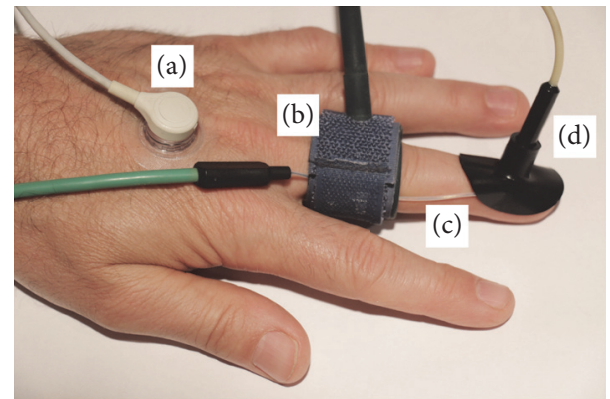

Figure 1: Position of the probes and measuring equipment on the hand: (a) $\mathrm{TcPO}_{2}$ probe, (b) pressure cuff, (c) thermometer, and (d) LDF probe and probe holder.

on the dorsal aspect of distal phalanx of the right middle finger at the level of the nail. The degree of adhesion of the probe to the skin was controlled by the flowmeter sensor. LDF measurements are expressed in arbitrary perfusion units (PU); thus the bMBF values were recorded in such units. Following baseline blood flow measurement, a small pressure cuff was mounted at the level of the proximal phalanx of the tested finger and inflated to $200 \mathrm{mmHg}$ to induce occlusion for a period of one minute, starting from the moment the biological zero flow signal is registered by the LDF machinery. The pressure was then released in the cuff within one second and the time to peak blood flow return was measured (tpMBF). Peak blood flow was considered as the highest recorded blood flow value assessed in PU after release of the pressure cuff. The LDF was measured for a period of 5 minutes after occlusion. A cuff inflation pressure of $200 \mathrm{mmHg}$ and an occlusion duration of one minute were chosen for the study based on recommendations of Bollinger and Fagrell, who described a one-minute arterial occlusion to be optimal for assessment of postocclusive reactive hyperemia in finger nailfold capillaries [42].

2.4. $\mathrm{TcPO}_{2}$. Transcutaneous oxygen tension measurements were made using a calibrated TCO2M (Novametrix Medical Systems Inc., Wallingford, USA) apparatus at an electrode temperature of $44^{\circ} \mathrm{C}, 20$ minutes after LDF testing of the tpMBF. The patient's skin was cleaned with alcohol and electrodes were positioned on the dorsum of the hand in the second intermetacarpal space. Transcutaneous oxygen tension levels were recorded after stabilization, over a period of 5 minutes.

2.5. Statistical Analysis. Statistical analysis was conducted using the SPSS Statistics 17.0 software package (SPSS Inc., Chicago, IL, USA) and MedCalc ver. 13.1.2.0 for Windows (MedCalc Software Inc., Marierke, Belgium). All data was tested for normality using the Shapiro-Wilks tests. The independent $t$-test was conducted on normally distributed data, while nonparametric testing using the Mann-Whitney $U$ test was employed on data that did not meet the criteria of normality. A $p$ value $<0.05$ was considered significant.

Receiver operating characteristic curve analysis was completed using the MedCalc software to evaluate diagnostic values of the tests. All diagnostic cut-off points were determined by computing Youden's index [43], a single statistic for assessing diagnostic performance, for each of the three tests. Based on the cut-off points, the sensitivity, specificity, positive predictive value, and negative predictive values were computed. For each ROC curve, the AUC was calculated. The AUC describes the chance of a randomly selected person with RP testing positive for the condition using one of the aforementioned tests, as compared to a randomly selected patient who does not have RP (an AUC of $1=$ a perfect test where all patients are with RP test positive for the disease using the selected test; $0.5=$ noninformative test).

2.6. Ethics. The protocol of this study was approved by the Jagiellonian University Medical College Bioethics Committee. Written, informed consent was given by each participant prior to inclusion into the study. This study has been conducted in accordance with the 1963 Helsinki Declaration and its later amendments.

\section{Results}

Baseline microvascular blood flow and tpMBF data were not normally distributed; thus nonparametric analysis using the Mann-Whitney $U$ test was employed, while the $\mathrm{TcPO}_{2}$ data was normally distributed allowing for an independent $t$-test to be conducted. Descriptive statistics for the study population can be found in Table 1. There was no significant difference in the mean age between controls $(23.12 \pm 2.38$ years $)$ and patients with RP $(24.97 \pm 6.37$ years; $p=0.43)$. Furthermore, no sex differences were found for any of the measures (all $p$ values $>0.05$ ). The mean duration of disease for patients with primary RP was $6.60( \pm 4.85)$ years.

The mean finger skin temperature of patients with RP after a 15-minute resting period in a temperature controlled room was $26.47( \pm 2.50)$ degrees Celsius, while the mean temperature for controls was $27.35( \pm 2.34 ; p=0.021)$ degrees Celsius. No significant correlation was found between mean finger skin temperature and any other variable for both the patient group and the control group.

Compared with controls, patients with RP had a lower bMBF $(15.2 \pm 6.45$ versus $24.7 \pm 9.36 \mathrm{PU}$; $U=1971 ; p<$ $0.001)$, higher tpMBF values $(16.4 \pm 10.7$ versus $4.87 \pm 1.75$ seconds; $U=787 ; p<0.001)$, and lower $\mathrm{TcPO}_{2}(56.2 \pm 9.73$ versus $66.7 \pm 10.8 \mathrm{mmHg} ; t=-7.24 ; p<0.001$; Figure 2). No significant difference in peak blood flow was found between the study group and the control group $(t=1.97 ; p=0.54)$. The mean peak blood flow for the study group was $84.1 \pm$ 23.2 PU and for the control group was $91.2 \pm 28.2 \mathrm{PU}$.

3.1. ROC Analysis. Cut-off values to diagnose primary RP for each diagnostic test were obtained by computing Youden's index. The calculated cut-offs used for bMBF, tpMBF, and $\mathrm{TcPO}_{2}$ were $\leq 19.3 \mathrm{PU},>7.2$ seconds, and $\leq 62.6 \mathrm{mmHg}$, respectively. Based on these cut-offs, the sensitivity, specificity, PPV, and NPV of each diagnostic test were computed (Table 2). Receiver operating characteristic curves generated for each diagnostic test can be found in Figure 3, along with the corresponding sensitivity and specificity 
TABLE 1: Descriptive values of the study population.

(a) Patient's diagnosed with Raynaud's syndrome

\begin{tabular}{lcccccc}
\hline & $N$ & Minimum & Maximum & Mean & Standard error & Standard deviation \\
\hline bMBF (PU) & 100 & 4.30 & 33.2 & 15.2 & 0.645 & 6.45 \\
tpMBF (seconds) & 100 & 3.00 & 65.0 & 16.4 & 1.070 & 10.7 \\
$\mathrm{TcPO}_{2}$ (mmHg) & 100 & 34.2 & 84.2 & 56.2 & 0.972 & 9.73 \\
\hline
\end{tabular}

(b) Controls

\begin{tabular}{lcccccc}
\hline & $N$ & Minimum & Maximum & Mean & Standard error & Standard deviation \\
\hline bMBF (PU) & 100 & 10.20 & 46.10 & 24.7 & 0.936 & 9.36 \\
tpBF (seconds) & 100 & 1.60 & 46.10 & 4.87 & 0.175 & 1.75 \\
$\mathrm{TcPO}_{2}$ (mmHg) & 100 & 45.90 & 91.10 & 66.7 & 1.080 & 10.8 \\
\hline
\end{tabular}
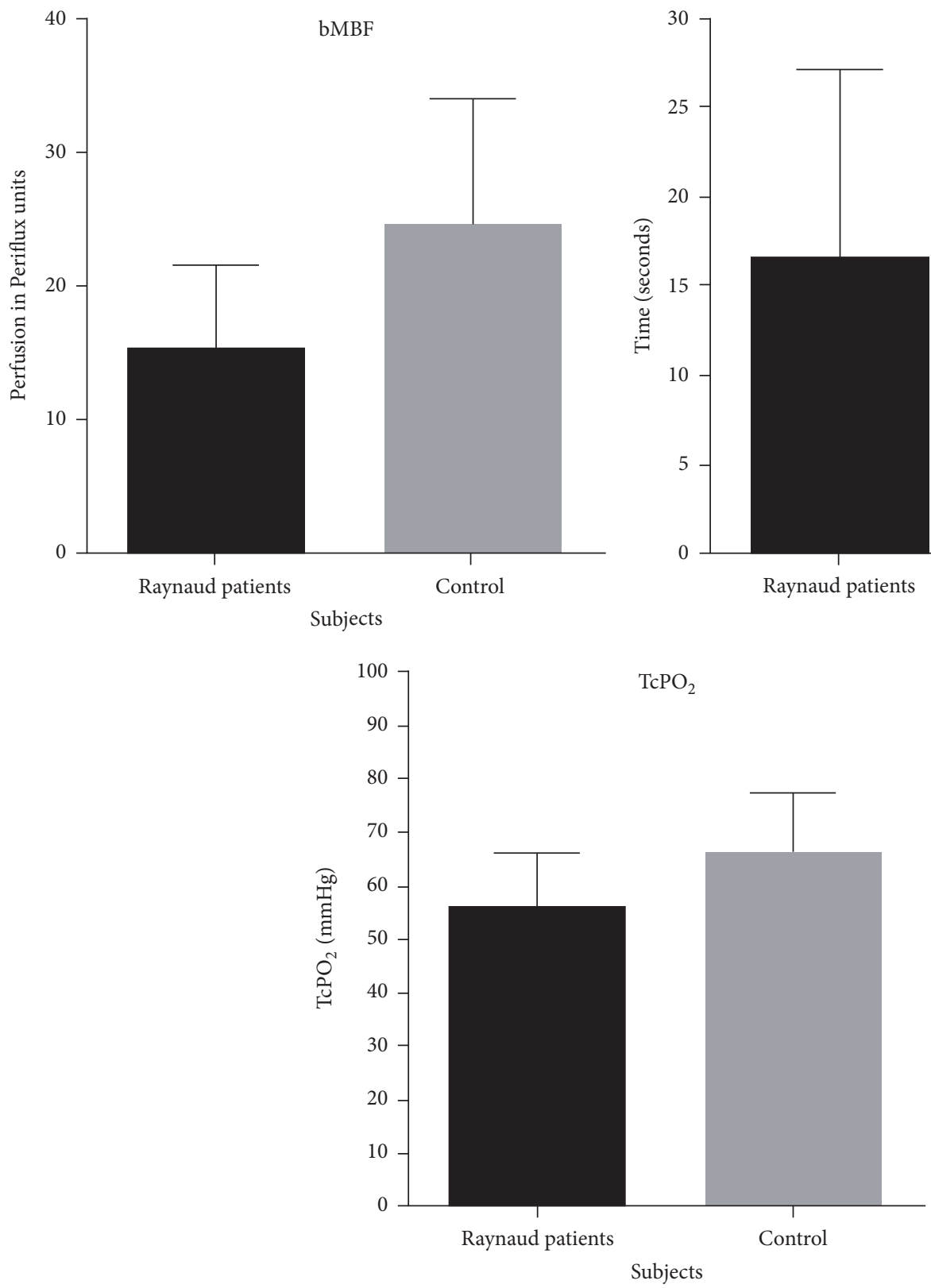

Figure 2: Difference in values obtained for bMBF, tpMBF, and $\mathrm{TcPO}_{2}$ between controls and patients with primary RP. bMBF: baseline microvascular blood flow; tpMBF: postocclusive time to peak microvascular blood flow; $\mathrm{Tc}_{\mathrm{CO}}$ : transcutaneous oxygen tension. 
TABLE 2: Sensitivity and specificity of the diagnostic tests.

\begin{tabular}{lccccccccc}
\hline & Cut-off value & Sensitivity & $95 \%$ CI & Specificity & $95 \%$ CI & PPV & $95 \%$ CI & NPV & $95 \%$ CI \\
\hline bMBF & $\leq 19.3 \mathrm{PU}$ & $79 \%$ & $69.7-86.5$ & $66 \%$ & $55.8-75.2$ & $69.9 \%$ & $60.6-78.2$ & $75.9 \%$ & $65.5-84.4$ \\
$\mathrm{TcPO}_{2}$ & $\leq 62.6 \mathrm{mmHg}$ & $77 \%$ & $67.5-84.8$ & $64 \%$ & $53.8-73.4$ & $68.1 \%$ & $58.8-76.6$ & $73.6 \%$ & $63.0-82.5$ \\
tpMBF & $>7.2$ seconds & $79 \%$ & $69.7-86.5$ & $90 \%$ & $82.4-95.1$ & $88.8 \%$ & $80.3-94.5$ & $81.1 \%$ & $72.6-87.9$ \\
\hline
\end{tabular}

PPV: positive predictive value; NPV: negative predictive value; $95 \%$ CI: $95 \%$ confidence interval.
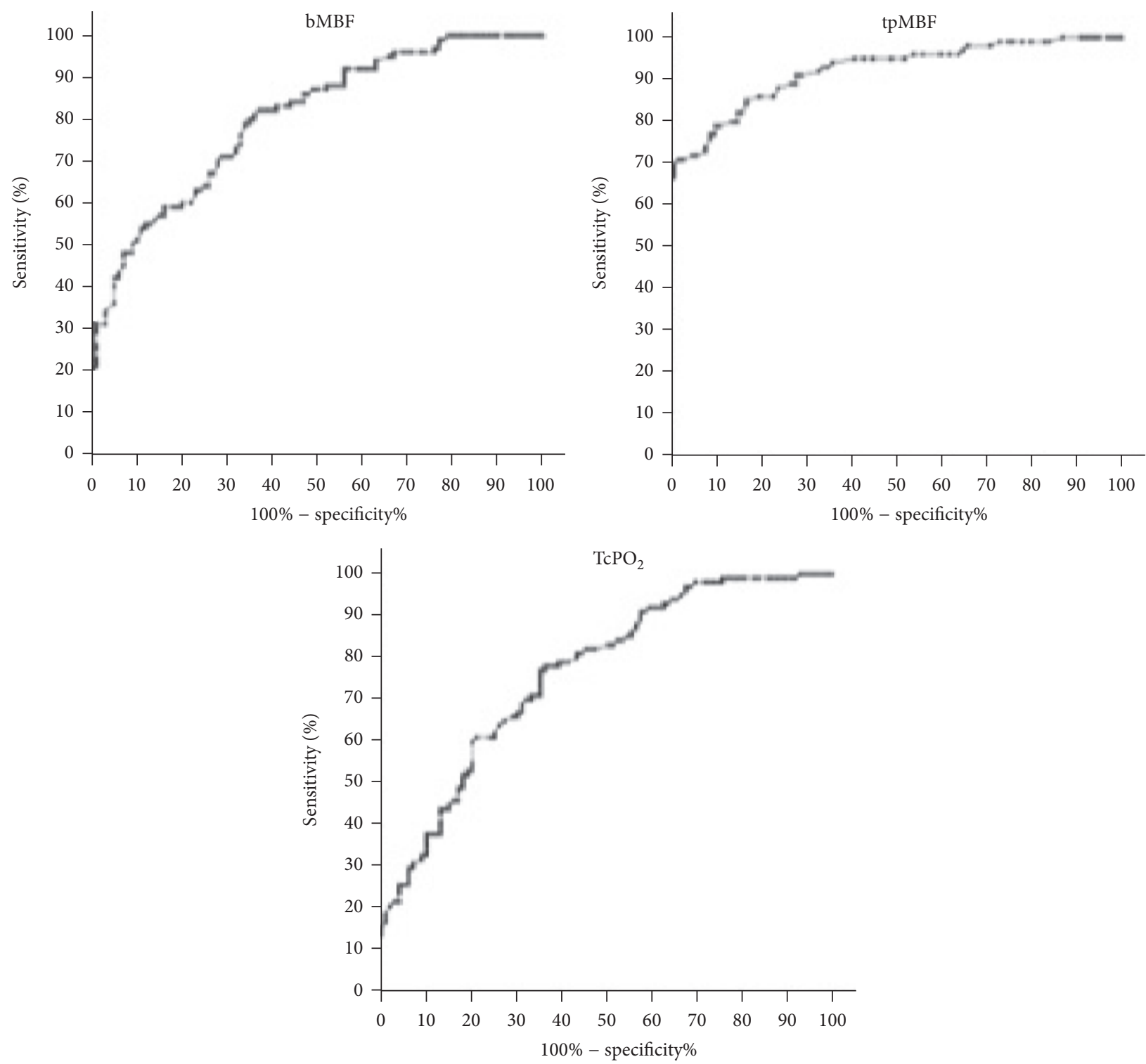

FIGURE 3: ROC curves of the predictive power of basal microvascular blood flow, LDF measures of time to max blood flow following vascular occlusion, and $\mathrm{TcPO}_{2}$ on the presence or absence of Raynaud's syndrome. bMBF: baseline microvascular blood flow; tpMBF: postocclusive time to peak microvascular blood flow; $\mathrm{TcPO}_{2}$ : transcutaneous oxygen tension.

plot versus criterion values which can be found in Supplement 1 in Supplementary Material available online at http://dx.doi.org/10.1155/2016/9645705.

The sensitivities of bMBF (79.0\%; 95\% CI: 69.7-86.5), $\mathrm{TcPO}_{2}$ (77.0\%; 95\% CI: 67.5-84.8), and tpMBF (79.0\%; 95\%
CI: 69.7-86.5) were all similar. However, tpMBF was found to have a significantly greater specificity $(90.0 \%$; $95 \%$ CI: $82.4-$ $95.1)$ as compared to bMBF $(66.0 \%$; $95 \%$ CI: $55.8-75.2)$ and $\mathrm{TcPO}_{2}$ (64.0\%; 95\% CI: 53.8-73.4), indicating that tpMBF is the superior test at ruling in the diagnosis of primary RP. 
TABLE 3: Area under the curve (AUC) for the three diagnostic techniques examined.

\begin{tabular}{lcccc}
\hline & AUC & SE & $95 \%$ CI & $p$ \\
\hline bMBF & 0.8029 & 0.0301 & $0.7439-0.8619$ & $<0.0001$ \\
tpMBF & 0.9212 & 0.0190 & $0.8840-0.9583$ & $<0.0001$ \\
$\mathrm{TcPO}_{2}$ & 0.7589 & 0.0334 & $0.6935-0.8213$ & $<0.0001$ \\
\hline
\end{tabular}

SE: standard error; CI: confidence interval.

This is also reflected in the greater positive likelihood ratio of tpMBF (7.9; 95\% CI: 4.4-14.3) as compared to bMBF (2.32; 95\% CI: $1.7-3.1)$ and $\mathrm{TcPO}_{2}(2.14 ; 95 \% \mathrm{CI}: 1.6-2.8)$. Negative likelihood ratios of tpMBF $(0.23$; 95\% CI: $0.2-0.3)$, bMBF (0.32; 95\% CI: $0.2-0.5)$, and $\mathrm{TcPO}_{2}$ (0.36; 95\% CI: $\left.0.2-0.5\right)$ were not significantly different.

The AUC values for the ROC curves are shown in Table 3. Pairwise comparison of the AUCs for the three diagnostic tests revealed that the AUC for the tpMBF plot was significantly different than both $\operatorname{bMBF}(z=3.33 ; p=0.0009)$ and $\mathrm{TcPO}_{2}(z=4.12 ; p<0.0001)$ measures. AUCs for $\mathrm{bMBF}$ and $\mathrm{TcPO}_{2}$ were not found to be significantly different $(p>0.05)$. The AUC of tpMBF (0.92; 95\% CI: $0.88-0.95)$ was greater than that of bMBF $(0.80 ; 95 \% \mathrm{CI}: 0.74-0.86)$ and $\mathrm{TcPO}_{2}(0.76 ; 95 \% \mathrm{CI}: 0.69-0.82)$, indicating the superior diagnostic accuracy of tpMBF for diagnosing primary RP as compared to the other measures. Additional data regarding the ROC curve analysis can be found in Supplement 2.

\section{Discussion}

Blood flow is controlled by a complex network of vasoconstrictive and vasodilative processes mediated by neural input and the release of vasoactive substances. In RP, the vasospastic attacks likely reflect changes in the balance of such physiological mechanisms [4, 44-46]. In the present investigation, bMBF and $\mathrm{TcPO}_{2}$ measures were significantly reduced in patients with primary RP as compared to controls. A previous study by Wollersheim et al. [22] also examined bMBF in patients with RP versus controls and found similar significant differences. The authors further investigated bMBF between patients with primary versus secondary RP but found no significant difference between these two groups [22].

Interestingly, though bMBF was significantly different between the groups in our study, peak blood flow after occlusion, measured in arbitrary perfusion units, was not significantly different between the study and control groups. However, while both patients with primary RP and controls can reach peak blood flows of similar magnitude, the time by which this occurs differentiates patients from controls.

In the posthyperemic time to peak blood flow measured by LDF, patients with RP took over 3 times longer to reach peak blood flow. This test had a greater specificity $(90.0 \%)$ and a stronger positive likelihood ratio (7.9) as compared to bMBF $(66.0 \%, 2.32)$ and $\mathrm{TcPO}_{2}(64.0 \%, 2.14)$ measures. As such, while sensitives were not significantly different among the three modalities, the higher specificity and positive likelihood ratio of tpMBF, as compared to bMBF and $\mathrm{Tc} \mathrm{PO}_{2}$, demonstrate tpMBF's superior ability to rule-in the diagnosis of primary RP.
Receiver operating characteristic curve analysis for the postocclusive time to peak blood flow (tpMBF) demonstrated that this diagnostic modality is highly accurate, with an AUC of 0.92. This indicates that a randomly selected person with RP is very likely to test positive (approximately 92\%) for the condition using the LDF postocclusion hyperemia measurement of time to peak blood flow. While less accurate than tpMBF, bMBF and $\mathrm{TcPO}_{2}$ also had AUC values that were fairly high (0.80 and 0.76 , resp.) and there was no significant difference between these two measures.

The advantage of postocclusion hyperemia measures in differentiating between cases and controls may stem from the provocation of a physiologic induced vasodilator response. Although the exact mechanism is unknown, reactive hyperemia is thought to occur due to the build-up of vasodilator metabolites such as endothelium-derived nitric oxide following vascular occlusion, which decreases vascular resistance leading to increased blood flow to the ischemic tissue [47, 48]. In patients with primary RP, it has been found that the dilator responses, induced by iontophoresis of the NO donor nitroprusside, are reduced in the digital vessels suggesting that the dilator action of $\mathrm{NO}$ is depressed in $\mathrm{RP}[45,46]$. This decrease in $\mathrm{NO}$ dilator function may in part be explained by the inactivation of $\mathrm{NO}$ by oxidative stress, stemming from repeated vasospastic episodes, followed by ischemiareperfusion [44].

Additionally, increased production of the potent endothelial derived vasoconstrictor endothelin-1, as well as a lack of downregulation of endothelin receptors, has been reported in patients with RP and may also help explain the observed prolonged time to postocclusive peak blood flow in RP patients as compared to controls $[49,50]$. However, other studies have found no difference in resting endothelin levels between patients with RP and controls [51].

Furthermore, it has been suggested that the cyclooxygenase (COX) pathway produces vasoconstrictive metabolites such as prostaglandin $\mathrm{H}_{2}$, which may limit finger vasodilation in RP patients [52]. Aspirin, a blocker of COX activity, was found to enhance vasodilation evoked by the iontophoresis of acetylcholine in RP [52]. These studies provide evidence to suggest that an imbalance between vasoconstriction and vasodilation is likely to be at least partially involved in the mechanism in RP.

The LDF measured time to peak blood flow following occlusion was best able to capture the differences between controls and cases, likely by forcing the ischemic digit to evoke the compensatory vasodilator response which may be limited in RP patients. Vasodilator mechanisms responsible for maintaining blood flow can affect both the bMBF and $\mathrm{TcPO}_{2}$ levels, but are more active under periods of deviation 
from homeostasis. This may have made the tpMBF measure more accurate at capturing the vascular response changes between controls and RP patients.

The lack of a standardized diagnostic test for RP limits the ability of clinicians to accurately diagnose the condition and instead forces them to rely solely on patient's history and clinical observations. Recently, a panel of international experts formulating a consensus on criteria for the diagnosis of RP scored LDF to be an inappropriate test for being both difficult to implement clinically and having a questionable utility [6]. However, we suggest that using LDF to measure the postocclusive time to peak blood flow may be a quick and easy to use noninvasive diagnostic test to increase the accuracy of the diagnosis of suspected primary RP in a patient in whom the clinical picture and laboratory results do not suggest an underlying secondary cause of the symptoms. With its high sensitivity and specificity, tpMBF may aid in objectively assessing patients with suspected primary RP, and future studies should further examine the clinical efficacy of this diagnostic modality.

However, to be universally applied as a diagnostic test in $\mathrm{RP}$, the test should have a high specificity for discriminating between primary and secondary RP [15]. In a similar study by Wollersheim et al. [22], the measurement of postocclusive recovery time by $\mathrm{LDF}$ was able to accurately discriminate between primary and secondary RP. However, further clinical studies are needed to assess the diagnostic value and efficacy of tpMBF to distinguish between primary and secondary RP, which was not measured in this study.

Some of the difficulties with implementation of LDF as a diagnostic tool are related to its single point of measure [35]. These include the sensitivity of the probe to tissue heterogeneity and movement artefacts. Some of the issues with implementation can be overcome through the use of dynamic testing, such as postocclusive reactive hyperemia, with a probe that is in a fixed position, firmly attached to skin [35]. Newer laser Doppler techniques, such as laser Doppler imaging (LDI), which measures flux over an area instead of a single point, are being explored for the use in patients with RP and have thus far reported interesting results [36-38]. However, the newer LDI equipment is more expensive and is not yet readily accessible for many clinicians [35].

In our study, significant differences were found in the mean finger skin temperature between patient's with RP and controls after a 15-minute resting period in a temperature controlled room. It was interesting to find that despite a slightly lower mean finger skin temperature, controls had a higher bMBF. However, it is important to note that tpMBF as measured by LDF is independent of skin temperature, and, moreover, there were no correlations between skin temperature and any other variable for either RP patients or controls.

The present study did not find any difference in the basal microvascular blood flow between men and women, which is in contrast to previous data that found basal cutaneous blood flow in the hands and fingers of young women to be roughly half of that seen in young men [53]. However, there was a predominance of females and a small sample of males, thus limiting the ability to detect such differences. This study also focused on single testing sessions which mimics clinical settings but limits the ability to detect changes in the results between sessions. Multiple testing sessions could help identify any variability in the results that could hinder the diagnostic value. Lastly, it would be worthwhile to identify the dominant hand and look at blood flow across both hands, as well as across all digits, to see how uniform the reported changes are.

Though newer integrating LDF probes decrease spatial variability and likely improve accuracy as compared to a single point LDF as used in this study, they are more expensive, with few clinics having access to such equipment to data. However, as costs decrease and integrating probes become more accessible to clinicians, these probes may be an improved alternative to single point LDF. In particular, LDI, due its ability to measure flux over an area, has the potential to improve accuracy over a single point LDF. Early studies applying this modality in the diagnosis of RP have shown promising results [36-38]. As such, future studies should assess the diagnostic utility of LDI in measuring postocclusive hyperemia.

\section{Conclusions}

Time to peak blood flow, as measured by LDF, is a quick, easy to use diagnostic tool that shows high sensitivity and specificity for differentiating patients with RP from healthy controls. As such, tpMBF may be used to objectively diagnose patients with primary RP, after exclusion of potential secondary causes of the condition. The efficacy of this diagnostic modality may lie in its ability to capture the deficiency in the occlusion induced vasodilator responses of the implicated vasculature.

\section{Competing Interests}

The authors declare that there is no conflict of interests.

\section{Acknowledgments}

This project was funded under the statutory funds of the Jagiellonian University Medical College. Krzysztof A. Tomaszewski was supported by the Foundation for Polish Science (FNP).

\section{References}

[1] M. Raynaud, On Local Asphyxia and Symmetrical Gangrene of the Extremities, E.Leclerc Paris, Paris, France, 1862.

[2] N. A. Flavahan, S. Flavahan, S. Mitra, and M. A. Chotani, "The vasculopathy of Raynaud's phenomenon and scleroderma," Rheumatic Disease Clinics of North America, vol. 29, no. 2, pp. 275-291, 2003.

[3] L. Fraenkel, "Raynaud's phenomenon: epidemiology and risk factors," Current Rheumatology Reports, vol. 4, no. 2, pp. 123128, 2002.

[4] F. Boin and F. M. Wigley, "Understanding, assessing and treating Raynaud's phenomenon," Current Opinion in Rheumatology, vol. 17, no. 6, pp. 752-760, 2005. 
[5] S. T. Valdovinos and G. J. Landry, "Raynaud syndrome," Techniques in Vascular and Interventional Radiology, vol. 17, no. 4, pp. 241-246, 2014.

[6] E. Maverakis, F. Patel, D. G. Kronenberg et al., "International consensus criteria for the diagnosis of Raynaud's phenomenon," Journal of Autoimmunity, vol. 48-49, pp. 60-65, 2014.

[7] F. M. Wigley, "Clinical practice. Raynaud's phenomenon," New England Journal of Medicine, vol. 347, no. 13, pp. 1001-1008, 2002.

[8] T. Tenland, E. G. Salerud, G. E. Nilsson et al., "Spatial and temporal variations in human skin blood flow," International Journal of Microcirculation. Clinical and Experimental, vol. 2, no. 2, pp. 81-90, 1983.

[9] R. De Angelis, F. Salaffi, and W. Grassi, "Health-related quality of life in primary raynaud phenomenon," Journal of Clinical Rheumatology, vol. 14, no. 4, pp. 206-210, 2008.

[10] M. Hughes, A. Snapir, J. Wilkinson, D. Snapir, F. M. Wigley, and A. L. Herrick, "Prediction and impact of attacks of Raynaud's phenomenon, as judged by patient perception," Rheumatology, vol. 54, no. 8, pp. 1443-1447, 2015.

[11] T. Kimura, S. Yoshizaki, N. Tsushima, M. Sano, and G. Hanai, "Buerger's colour," British Journal of Surgery, vol. 77, no. 11, pp. 1299-1301, 1990.

[12] K. Yamamoto, S. Iwase, T. Mano, and S. Shionoya, "Muscle sympathetic outflow in Buerger's disease," Journal of the Autonomic Nervous System, vol. 44, no. 1, pp. 67-75, 1993.

[13] P. Lee, J. Sarkozi, A. A. Bookman, E. C. Keystone, and S. K. Armstrong, "Digital blood flow and nailfold capillary microscopy in Raynaud's phenomenon," Journal of Rheumatology, vol. 13, no. 3, pp. 564-569, 1986.

[14] E. Mannarino, L. Pasqualini, F. Fedeli, V. Scricciolo, and S. Innocente, "Nailfold capillaroscopy in the screening and diagnosis of Raynaud's phenomenon," Angiology, vol. 45, no. 1, pp. 37-42, 1994.

[15] M. E. Anderson, T. L. Moore, M. Lunt, and A. L. Herrick, “The 'distal-dorsal difference': a thermographic parameter by which to differentiate between primary and secondary Raynaud's phenomenon," Rheumatology, vol. 46, no. 3, pp. 533-538, 2007.

[16] J. D. Pauling, V. Flower, J. A. Shipley, N. D. Harris, and N. J. McHugh, "Influence of the cold challenge on the discriminatory capacity of the digital distal-dorsal difference in the thermographic assessment of Raynaud's phenomenon," Microvascular Research, vol. 82, no. 3, pp. 364-368, 2011.

[17] M. Vayssairat, N. Baudot, N. Blaison et al., "Limitations of cold tests in Raynaud's disease," Journal des Maladies Vasculaires, vol. 15, no. 1, pp. 82-85, 1990.

[18] H. Cai, H. Pettersson, H. Rohman, S.-E. Larsson, and P. Å. Öberg, "A new single-fibre laser Doppler flowmeter based on digital signal processing," Medical Engineering and Physics, vol. 18, no. 7, pp. 523-528, 1996.

[19] E. Del Bianco, B. Magini, G. Muscarella, P. Cappugi, and T. Lotti, "Raynaud's phenomenon (primary or secondary to systemic sclerosis). The usefulness of laser-doppler flowmetry in the diagnosis," International Angiology, vol. 20, no. 4, pp. 307-313, 2001.

[20] P. A. Oberg, T. Tenland, and G. E. Nilsson, "Laser-Doppler flowmetry-a non-invasive and continuous method for blood flow evaluation in microvascular studies," Acta Medica Scandinavica, vol. 216, no. 687, pp. 17-24, 1984.

[21] Z. Stoyneva, "Laser Doppler-recorded venoarteriolar reflex in Raynaud's phenomenon," Autonomic Neuroscience: Basic and Clinical, vol. 116, no. 1-2, pp. 62-68, 2004.
[22] H. Wollersheim, J. Reyenga, and T. Thien, "Postocclusive reactive hyperemia of fingertips, monitored by laser doppler velocimetry in the diagnosis of Raynaud's phenomenon," Microvascular Research, vol. 38, no. 3, pp. 286-295, 1989.

[23] M. J. Lim, S. R. Kwon, K.-H. Jung, K. Joo, S.-G. Park, and W. Park, "Digital thermography of the fingers and toes in Raynaud's phenomenon," Journal of Korean Medical Science, vol. 29, no. 4, pp. 502-506, 2014.

[24] O. Schlager, M. E. Gschwandtner, K. Herberg et al., "Correlation of infrared thermography and skin perfusion in Raynaud patients and in healthy controls," Microvascular Research, vol. 80, no. 1, pp. 54-57, 2010.

[25] R. Inaba, M. Ariizumi, T. Furuno, M. Tabata, and A. Okada, "Changes in the forearm tcPO2 following the cold water immersion test," Sangyo Igaku, vol. 28, no. 4, pp. 279-282, 1986.

[26] J. L. Silverstein, V. D. Steen, T. A. Medsger Jr., and V. Falanga, "Cutaneous hypoxia in patients with systemic sclerosis (scleroderma)," Archives of Dermatology, vol. 124, no. 9, pp. 1379-1382, 1988.

[27] H. Wollersheim and T. Thien, "Transcutaneous pO2 measurements in Raynaud's phenomenon. Value and limitations," International Journal of Microcirculation, Clinical and Experimental, vol. 7, no. 4, pp. 357-366, 1988.

[28] T. Kanetaka, T. Komiyama, A. Onozuka, T. Miyata, and H. Shigematsu, "Laser Doppler skin perfusion pressure in the assessment of Raynaud's phenomenon," European Journal of Vascular and Endovascular Surgery, vol. 27, no. 4, pp. 414-416, 2004.

[29] O. Bongard and B. Fagrell, "Variations in laser Doppler flux and flow motion patterns in the dorsal skin of the human foot," Microvascular Research, vol. 39, no. 2, pp. 212-222, 1990.

[30] G. A. Nilsson, T. Tenland, and P. Å. Öberg, "Evaluation of a laser doppler flowmeter foi measurement of tissue blood flow," IEEE Transactions on Biomedical Engineering, vol. 27, no. 10, pp. 597604, 1980.

[31] S. Sundberg, "Acute effects and long-term variations in skin blood flow measured with laser Doppler flowmetry," Scandinavian Journal of Clinical and Laboratory Investigation, vol. 44, no. 4, pp. 341-345, 1984.

[32] T. Tenland, E. G. Salerud, G. E. Nilsson, and P. A. Oberg, "Spatial and temporal variations in human skin blood flow," International journal of microcirculation, clinical and experimental/sponsored by the European Society for Microcirculation, vol. 2, no. 2, pp. 81-90, 1983.

[33] N. E. Almond, D. P. Jones, and E. D. Cooke, "Noninvasive measurement of the human peripheral circulation: relationship between laser Doppler flowmeter and photoplethysmograph signals from the finger," Angiology, vol. 39, no. 9, pp. 819-829, 1988.

[34] G. Yosipovitch, J. Schneiderman, D. J. van Dyk, A. Chetrit, G. Milo, and G. Boner, "Impairment of the postural venoarteriolar response in young type 1 diabetic patients. A study by laser Doppler flowmetry," Angiology, vol. 47, no. 7, pp. 687-691, 1996.

[35] A. L. Herrick and S. Clark, "Quantifying digital vascular disease in patients with primary Raynaud's phenomenon and systemic sclerosis," Annals of the Rheumatic Diseases, vol. 57, no. 2, pp. 70-78, 1998.

[36] M. J. U. Correa, L. E. C. Andrade, and C. Kayser, "Comparison of laser Doppler imaging, fingertip lacticemy test, and nailfold capillaroscopy for assessment of digital microcirculation in systemic sclerosis," Arthritis Research and Therapy, vol. 12, article R157, 2010. 
[37] A. K. Murray, T. L. Moore, J. B. Manning, C. Taylor, C. E. M. Griffiths, and A. L. Herrick, "Noninvasive imaging techniques in the assessment of scleroderma spectrum disorders," Arthritis Care and Research, vol. 61, no. 8, pp. 1103-1111, 2009.

[38] E. Rosato, F. Borghese, S. Pisarri, and F. Salsano, "Laser Doppler perfusion imaging is useful in the study of Raynaud's phenomenon and improves the capillaroscopic diagnosis," The Journal of Rheumatology, vol. 36, no. 10, pp. 2257-2263, 2009.

[39] M. Vayssairat, J. F. Mathieu, P. Priollet, P. Vellay, J. C. Pines, and E. Housset, "Transcutaneous measurement of partial oxygen pressure. A new functional exploratory method in vascular pathology," Presse Medicale, vol. 13, no. 27, pp. 1683-1686, 1984.

[40] E. C. LeRoy and T. A. Medsger, "Raynaud's phenomenon: a proposal for classification," Clinical and Experimental Rheumatology, vol. 10, no. 5, pp. 485-488, 1992.

[41] S. N. Lambova and U. Müller-Ladner, "The role of capillaroscopy in differentiation of primary and secondary Raynaud's phenomenon in rheumatic diseases: a review of the literature and two case reports," Rheumatology International, vol. 29, no. 11, pp. 1263-1271, 2009.

[42] A. Bollinger and B. Fagrell, Clinical Capillaroscopy: A Guide for Its Use in Clinical Research and Practice, Hogrefe \& Huber Publishers, 1990.

[43] W. J. Youden, "Index for rating diagnostic tests," Cancer, vol. 3, no. 1, pp. 32-35, 1950.

[44] J. P. Cooke and J. M. Marshall, "Mechanisms of Raynaud's disease," Vascular Medicine, vol. 10, no. 4, pp. 293-307, 2005.

[45] C. M. Edwards and J. M. Marshall, "Cutaneous vasodilator responses to sodium nitroprusside but not those to acetylcholine may be impaired in patients with Primary Raynaud's Disease," Clinical Autonomic Research, vol. 9, pp. 35-35, 1999.

[46] S. J. Morris and A. C. Shore, "Skin blood flow responses to the iontophoresis of acetylcholine and sodium nitroprusside in man: possible mechanisms," Journal of Physiology, vol. 496, no. 2, pp. 531-542, 1996.

[47] A. Kilbom and A. Wennmalm, "Endogenous prostaglandins as local regulators of blood flow in man: effect of indomethacin on reactive and functional hyperaemia," Journal of Physiology, vol. 257, no. 1, pp. 109-121, 1976.

[48] J. Nowak and A. Wennmalm, "A study on the role of endogenous prostaglandins in the development of exercise-induced and post-occlusive hyperemia in human limbs," Acta Physiologica Scandinavica, vol. 106, no. 3, pp. 365-369, 1979.

[49] G. A. Knock, G. Terenghi, C. B. Bunker, H. A. Bull, P. M. Dowd, and J. M. Polak, "Characterization of endothelin-binding sites in human skin and their regulation in primary Raynaud's phenomenon and systemic sclerosis," Journal of Investigative Dermatology, vol. 101, no. 1, pp. 73-78, 1993.

[50] M. R. Zamora, R. F. O'Brien, R. B. Rutherford, and J. V. Weil, "Serum endothelin-1 concentrations and cold provocation in primary Raynaud's phenomenon," The Lancet, vol. 336, no. 8724, pp. 1144-1147, 1990.

[51] W. Bottomley and M. Goodfield, "A pathogenic role for endothelin in Raynaud's phenomenon?" Acta DermatoVenereologica, vol. 74, no. 6, pp. 433-434, 1994.

[52] M. J. Easter and J. M. Marshall, "Contribution of prostanoids to endothelium-dependent vasodilatation in the digital circulation of women with primary Raynaud's disease," Clinical Science, vol. 109, no. 1, pp. 45-54, 2005.

[53] J. P. Cooke, M. A. Creager, P. J. Osmundson, and J. T. Shepherd, "Sex differences in control of cutaneous blood flow," Circulation, vol. 82, no. 5, pp. 1607-1615, 1990. 


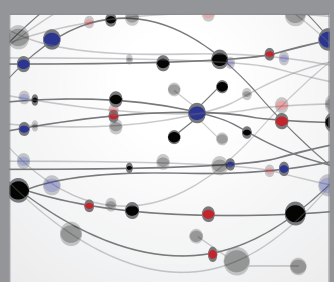

The Scientific World Journal
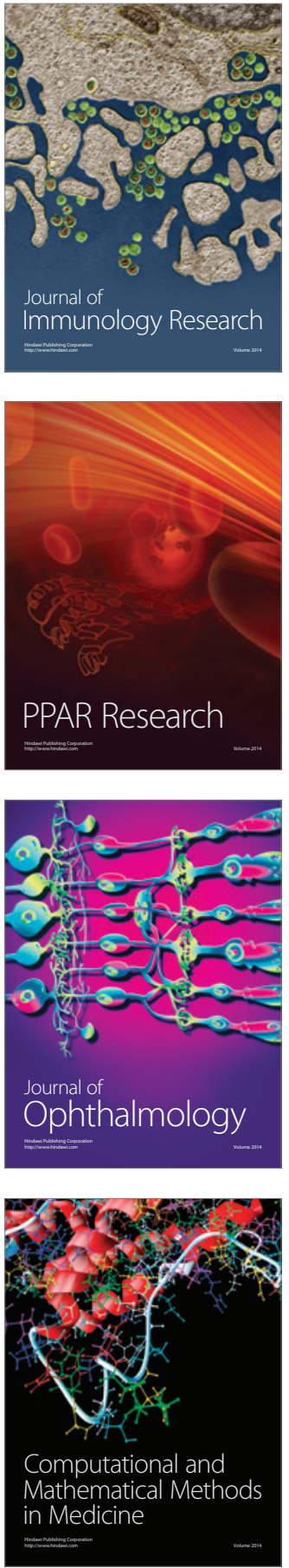

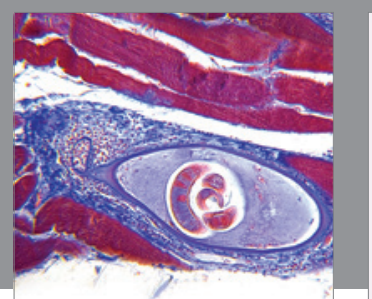

Gastroenterology Research and Practice

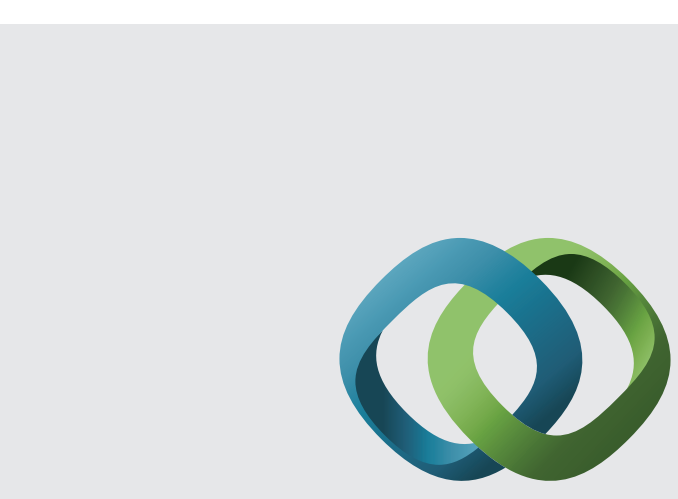

\section{Hindawi}

Submit your manuscripts at

http://www.hindawi.com
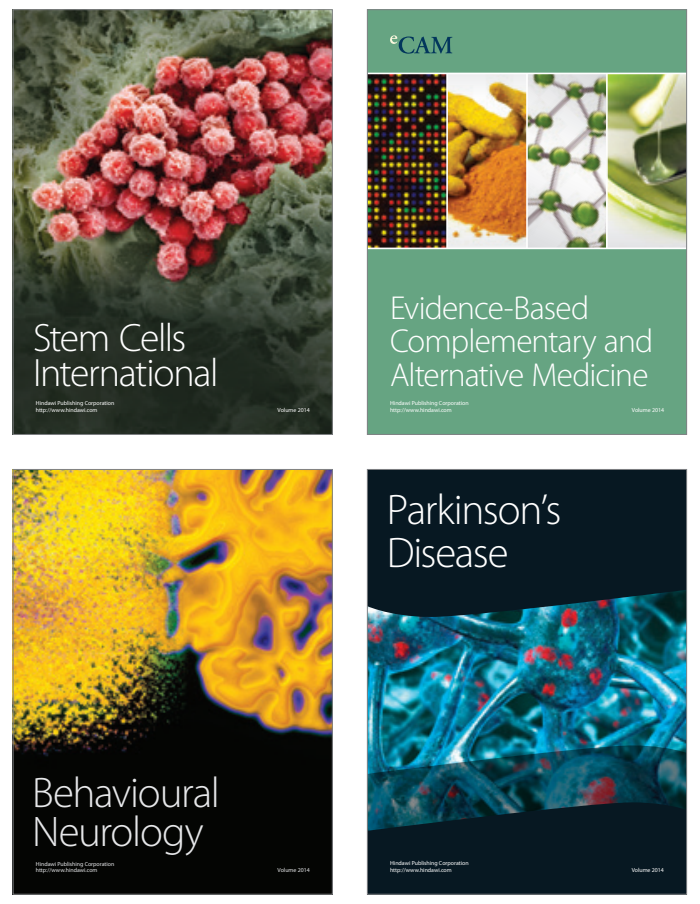
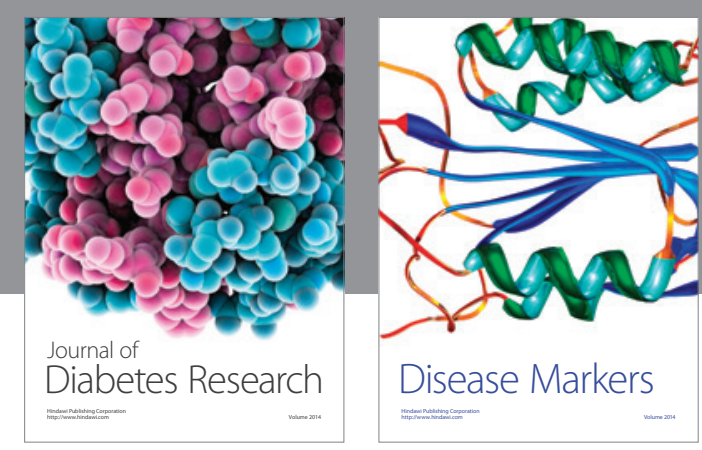

Disease Markers
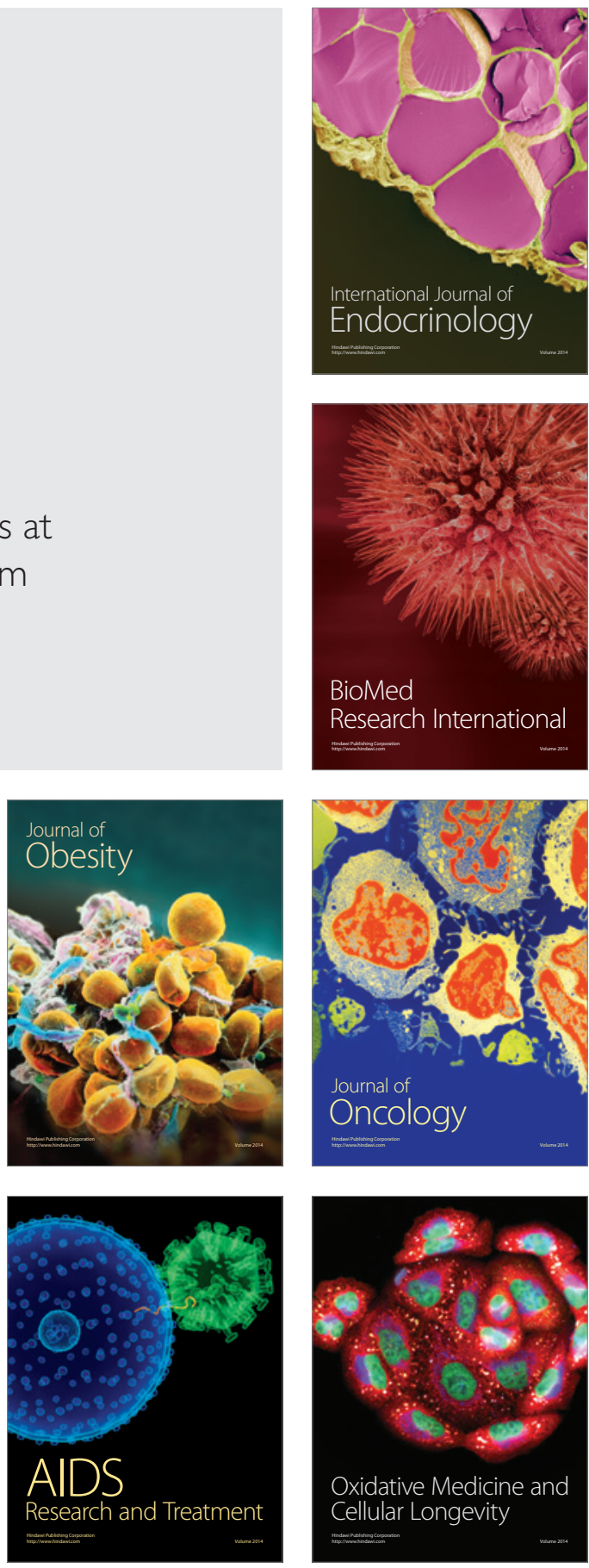